\title{
Romanian Translation and Cross-Cultural Adaptation of the SarQol Questionnaire
}

\author{
Gasparik Andrea Ildiko1, Mihai Gabriela2*, Pașcanu lonela Maria,3 \\ ${ }^{1}$ Public Health and Health Management Department of University of Medicine and Pharmacy Tîrgu Mures, Romania \\ 2 Mures County Hospital, Tîrgu Mures, Romania \\ 3 Endocrinology Department of University of Medicine and Pharmacy Tîrgu Mures, Romania
}

Sarcopenia, or age-related muscle loss is emerging as a major public health concern. A reduced quality of life (QoL) due to impaired physical performance associated with this disease has been evidenced in these individuals. Generic instruments, such as Short Form 36 questionnaire (SF-36), do not accurately assess the impact of sarcopenia on QoL. SarQol (Sarcopenia Quality of Life) questionnaire, was the first diseasespecific questionnaire addressing quality of life in patients with sarcopenia and has been recently designed for providing a global picture on quality of life in community-dwelling elderly subjects aged 65 years and older. Our aim was the translation and cultural adaptation of the original SarQol, to finally obtain a highly reliable instrument for the assessment of the quality of life of Romanian patients, affected by sarcopenia. We followed the recommended process, the international protocol of translation. The pretest process involved 20 subjects (10 sarcopenic and 10 non sarcopenic with different educational and socioeconomic backgrounds) who were asked to complete the questionnaire. Feedbacks were requested from all subjects regarding the clearness of questions, difficulties in completing the test or understanding the meaning of questions. Using the recommended best practice protocol for translation, the pre-final version is comparable with the original instrument in terms of content and accuracy. After the validation of psychometric properties, it should be a useful tool to assess Quality of Life and sarcopenia among elderly romanian patients.

Keywords: Quality of life; Questionnaire; Sarcopenia; SarQol

Received: 29 June 2016 / Accepted: 03 July 2016

\section{To the editor:}

Sarcopenia,or age-related muscle loss is emerging as a major public health concern. The muscle tissue is progressively lost with advancing age, process that results in diminished muscle mass and strength, this condition being referred to as sarcopenia [1]. A gradual deterioration in quality of life (QoL) has been evidenced in these individuals. However, much of this research assessing QoL in sarcopenia has been done using generic tools, such as Short Form 36 questionnaire (SF-36), which may not be ideal to accurately assess the impact of sarcopenia on QoL, hence the need for a specific instrument [2].

SarQol (Sarcopenia Quality of Life) questionnaire was the first disease-specific questionnaire addressing quality of life in patients with sarcopenia and has been recently designed for providing a global picture on quality of life in community-dwelling elderly subjects aged 65 years and older. It was developed initially in French language and has been translated and validated in English language one year later $[3,4]$.

Our aim was the translation and cultural adaptation of the original SarQol, to finally obtain a highly reliable instrument for the assessment of the quality of life of Romanian patients, affected by sarcopenia.

The original SarQol is a multidimensional questionnaire consisting in 55 items, translated into 22 questions which have been rated on a 4-point Likert scale. The 22 ques-

* Correspondence to: Gabriela Mihai

E-mail: mihaigabriela430@yahoo.com tions are distributed according to their relation with seven domains of dysfunction: Physical and mental health, Locomotion, Body composition, Functionality, Activities of daily living, Leisure activities and Fears [3].

We followed the recommended process, the international protocol of translation. The pretest process involved 20 subjects (10 sarcopenic and 10 non sarcopenic with different educational and socioeconomic backgrounds) who were asked to complete the questionnaire. Half of the subjects completed SarQol questionnaires in our presence, in order to directly assess possible perception difficulties. Feedbacks were requested from all subjects regarding the clearness of questions, difficulties in completing the test or understanding the meaning of questions. According to our assessment and feedbacks, in order to have literal but also conceptual equivalence, we modified 2 items, which were re-checked then with the linguist and the other committee members.

SarQol is the first specific quality of life questionnaire developed for sarcopenia, and without special challenges, we developed the translated, adapted and pre-validated version of the original SarQol questionnaire. The romanian SarQol version can be found at www.aspor.ro

Using the recommended best practice protocol for translation, the pre-final version is comparable with the original instrument in terms of content and accuracy.After the validation of psychometric properties, it should be a useful tool to assess Quality of Life and sarcopenia among elderly romanian patients. 


\section{References}

1. Marcell T. Review Article: Sarcopenia: Causes, Consequences, and Preventions. J Gerontol A Biol Sci Med Sci. 2003;58(10):M911-M916. doi: 10.1093/gerona/58.10.M911

2. Rizzoli R1, Reginster JY, Arnal JF, et al. Quality of life in sarcopenia and frailty. Calcif Tissue Int. 2013;93(2):101-20. doi: 10.1007/s00223-0139758-y.
3. Beaudart C, Biver E, Reginster JY, et al. Development of a selfadministrated quality of life questionnaire for sarcopenia in elderly subjects: the SarQoL. Age and Aging, 2015;44(6):960-966. doi: 10.1093/ ageing/afv133

4. Beaudart C, Edwards M, Moss C, et al. English translation and crosscultural adaptation of the SARQOL Questionnaire. Journal of Frailty \& Aging 2016;5(Supplement 1):58 